\title{
Evaluation of pharmacokinetics, user handling, and tolerability of peginterferon alfa-2a (40 kDa) delivered via a disposable autoinjector device
}

\author{
Peter Varunok' \\ Eric Lawitz ${ }^{2}$ \\ Kimberly L Beavers ${ }^{3}$ \\ Gary Matusow ${ }^{4}$ \\ Ruby Leong 5 \\ Nathalie Lambert ${ }^{6}$ \\ Coen Bernaards ${ }^{7}$ \\ Jonathan Solsky ${ }^{5}$ \\ Barbara J Brennan ${ }^{5}$ \\ Cynthia Wat ${ }^{8}$ \\ Anne Bertasso 5 \\ 'Gastroenterology Associates, \\ Poughkeepsie, NY, USA; \\ ${ }^{2}$ Alamo Medical Research, San \\ Antonio, TX, USA; ${ }^{3}$ Asheville \\ Gastroenterology, Asheville, NC, \\ USA; ${ }^{4}$ Gastroenterology Group, South \\ Jersey, NJ, USA; ${ }^{5}$ Roche, Nutley, NJ, \\ USA; ${ }^{6}$ Roche, Basel, Switzerland; \\ ${ }^{7}$ Roche, San Francisco, CA, USA; \\ ${ }^{8}$ Roche, Welwyn, UK
}

This article was published in the following Dove Press journal:

Patient Preference and Adherence

23 November 201I

Number of times this article has been viewed

Background: Peginterferon alfa-2a (40 kDa) is currently administered using a prefilled syringe. The peginterferon alfa-2a disposable autoinjector is a new safety-engineered device designed to facilitate injection and reduce the risk of needlestick injuries. The analysis of two open-label Phase I trials evaluated the pharmacokinetics, successful administration, and tolerability of peginterferon alfa-2a when using the autoinjector. The studies were performed to support the filing and registration of the autoinjector device.

Methods: In trial 1, 50 healthy adult subjects received one $180 \mu \mathrm{g}$ dose of peginterferon alfa- $2 \mathrm{a}$ via the autoinjector. Serial blood samples were collected predose, up to 336 hours following drug administration, and at follow-up ( $28 \pm 3$ days post-dosing) for noncompartmental pharmacokinetic analysis. Trial 2 randomized 60 adult patients with chronic hepatitis $\mathrm{C}$ to $180 \mu \mathrm{g}$ peginterferon alfa-2a once weekly by the autoinjector or prefilled syringe for 3 weeks followed by the alternative device (prefilled syringe or autoinjector, respectively) for 3 weeks. Patients also received ribavirin. Administration by the devices was evaluated under direct observation by a study staff member and by patient subjective assessment.

Results: In trial 1, following a single dose of peginterferon alfa-2a, the maximum plasma concentration was $16.1 \pm 5.3 \mathrm{ng} / \mathrm{mL}$ (mean \pm standard deviation), and area under the concentration time curve (0-168 hours) was $1996 \pm 613 \mathrm{ng} \cdot \mathrm{hour} / \mathrm{mL}$, similar to that reported using a vial/syringe or prefilled syringe. In trial 2, few patients showed handling difficulties with either device. Generally, patients were observed to be more satisfied and confident, followed instructions better, and successfully initiated injection with the autoinjector versus the prefilled syringe. Patients reported the autoinjector to be more convenient and easier to use. No pain or discomfort was experienced using the autoinjector. The autoinjector safety profile was consistent with that known for peginterferon alfa-2a/ribavirin.

Conclusion: These results indicate that peginterferon alfa-2a can be successfully and safely delivered via the autoinjector and that the device is easy to handle.

Keywords: peginterferon alfa-2a, disposable autoinjector, hepatitis $\mathrm{C}$, pharmacokinetics, user handling

\section{Introduction}

Peginterferon alfa-2a (40 kDa) is a polyethylene glycol (peg)-modified form of human recombinant interferon alfa-2a. Peginterferon alfa-2a, at a dose of $180 \mu \mathrm{g}$ once weekly, alone or in combination with ribavirin, is indicated for the treatment of chronic hepatitis $\mathrm{C}$ infection, including in patients with compensated cirrhosis, as well as coinfection with human immunodeficiency virus (HIV). ${ }^{1}$ It is also approved for the treatment of chronic hepatitis B infection. ${ }^{2}$ 
The current standard of care for hepatitis $\mathrm{C}$ is peginterferon alfa (peginterferon alfa-2a [40 kDa] or peginterferon alfa- $2 \mathrm{~b}$ [12 kDa]) plus ribavirin. The two peginterferon drugs have different pharmacologic properties and have different formulations and devices for administration. ${ }^{3-6}$ Peginterferon alfa-2a is currently licensed for administration via either a vial and syringe or a prefilled syringe. ${ }^{7}$ Both of these modes of injection employ a separate needle covered by a safety shield which has to be attached to the syringe; the safety shield must be manipulated to appropriate positions prior to and after use. There are a number of potential disadvantages to using these two devices which can impact on patient acceptance of treatment, including increased anxiety among patients with a fear of needles, the possibility of recidivism in former users of illicit injectable drugs, and the increased risk of needlestick injuries in noninfected individuals. It is estimated that, every year, one out of every seven health care professionals is accidentally injured by a contaminated sharp object, most often a needle, and approximately $62 \%-88 \%$ of such cases can potentially be prevented by the use of safer devices. ${ }^{8}$ Performing regular self-injections can also be mentally and physically stressful for many patients, and concerns regarding correct injection technique, pain on injection, and perceived lack of efficacy can be further potential issues affecting treatment acceptance. ${ }^{9,10}$

To address the above issues, a single-use, disposable autoinjector (prefilled pen) has undergone clinical development by the manufacturer of peginterferon alfa-2a (Roche, Basel, Switzerland). This device has features that help simplify the injection process and may help improve patient satisfaction and overcome injection-related issues. Using the autoinjector device, peginterferon alfa-2a is administered subcutaneously by pressing and releasing a spring-based activation button. After completion of the injection, the autoinjector is removed from the skin and the needle is automatically covered by the needle shield which locks in place. This is designed to limit needle exposure, thereby minimizing the risk for needlestick injuries, as well as to shield the needle from view and thus may reduce anxiety and the risk of recidivism, if applicable, among patients.

Disposable autoinjector combination products are already currently marketed for several drug products (eg, the Epipen ${ }^{\circledR}$ for anaphylaxis, the Rebiject ${ }^{\circledR}$ for multiple sclerosis, and the SureClick ${ }^{\circledR}$ for rheumatoid arthritis and anemia). Current evidence suggests that patients prefer autoinjector devices over manual injection via a prefilled syringe for drug administration, and patients have been reported to experience less pain with an autoinjector, and to perceive the device as easier and more convenient to use than a prefilled syringe. ${ }^{11-14}$ In addition, patient adherence to treatment regimens has been shown to be higher among patients using autoinjectors compared with a vial and syringe which may be based primarily on patient preference. ${ }^{15,16}$ This in turn may be driven by greater convenience, flexibility, and perceived clinical efficacy with autoinjector devices. ${ }^{17}$

Here, the results from two separate Phase I studies evaluating the peginterferon alfa-2a autoinjector device are presented. These studies were performed to support the filing and registration of the autoinjector device, and the design of the user handling protocol was reviewed and agreed to by the European and US health authorities. The first trial characterizes the pharmacokinetics and evaluates the safety profile and tolerability of a single $180 \mu \mathrm{g}$ dose of peginterferon alfa-2a when administered via a disposable autoinjector in healthy subjects. The second trial evaluates and compares the successful administration and tolerability of peginterferon alfa-2a using the autoinjector and the prefilled syringe in patients with chronic hepatitis $\mathrm{C}$.

\section{Methods}

Both studies were conducted in accordance with the principles of the Declaration of Helsinki or with the laws and regulations of the country in which the research was conducted. The studies fully adhered to the principles outlined in the current International Council on Harmonisation Guidelines for Good Clinical Practice or with local law if it afforded greater protection to the patient. Both study protocols were approved by the Institutional Review Board of each participating institution and written informed consent was obtained from all patients prior to any screening assessments.

\section{Trial I: pharmacokinetic study in healthy subjects}

\section{Subjects}

Subjects (aged 18-65 years, body mass index $\geq 18-\leq 30 \mathrm{~kg}$ / $\mathrm{m}^{2}$ ) were assessed as healthy volunteers, after having been medically examined and clinically tested. Complete blood count, blood pressure, heart rate, and electrocardiogram were normal, and HIV, hepatitis B and C, and pregnancy screenings (for women) were negative. Eligible patients were required to be nonsmokers (for at least 3 months prior to dosing), have a negative test for drugs of abuse and alcohol prior to dosing, and to have not received interferon or peginterferon within 8 weeks prior to dosing. Female subjects had to be surgically sterile or postmenopausal for the past year (amenorrhea for at least 12 consecutive months), and male subjects had to be 
willing to use one effective method of contraception during the study and for 90 days after drug administration.

The main exclusion criteria included the following: a history of drug or alcohol abuse within the last year; a history of significant allergic conditions; hypersensitivity to paracetamol; autoimmune, dermatologic, gastrointestinal, renal, hepatic, pulmonary, cardiac, hematologic, endocrine, neurologic, or clinically significant cardiovascular or cerebrovascular disease; a history of thyroid disease, severe retinopathy or significant psychiatric disease; use, or anticipated use, of any medications within 2 weeks or 5 half-lives of the drug before dosing; flu or cold-related symptoms within the 2 weeks prior to dosing or any self-limited disease, such as gastritis; and aspartate aminotransferase or alanine aminotransferase values at screening above the upper limit of normal.

\section{Study design}

The pharmacokinetic study (Trial 1) was an open-label, single-center, single-dose study conducted in France, in which 50 healthy subjects received one $180 \mu \mathrm{g} / 0.5 \mathrm{~mL}$ dose of peginterferon alfa-2a (Pegasys ${ }^{\circledR}$, Roche, Basel, Switzerland) administered subcutaneously by investigational staff using the disposable autoinjector. Serial blood samples were collected predose, up to 336 hours following drug administration, and at follow-up ( $29 \pm 3$ days after dosing) for noncompartmental pharmacokinetic analysis.

\section{Assessments}

The primary objective of this trial was to characterize the pharmacokinetics of a single $180 \mu \mathrm{g}$ dose of peginterferon alfa-2a when administered via an autoinjector. The secondary objective was to evaluate the safety profile and tolerability of peginterferon alfa-2a in healthy subjects. The pharmacokinetic parameters evaluated for each subject following peginterferon alfa-2a autoinjector administration were: observed maximum serum concentration $\left(\mathrm{C}_{\max }\right)$; time to $\mathrm{C}_{\max }$ $\left(\mathrm{T}_{\max }\right)$; area under the concentration-time curve (AUC) from time zero to the last measurable concentration $\left(\mathrm{AUC}_{0-\text { last }}\right)$ and $\mathrm{AUC}$ from time zero extrapolated to infinity $\left(\mathrm{AUC}_{0-\infty}\right)$; terminal elimination half-life $\left(\mathrm{t}_{1 / 2}\right)$; apparent volume of distribution $\left(\mathrm{V}_{\mathrm{z}} / \mathrm{F}\right)$; and apparent total body clearance $(\mathrm{Cl} / \mathrm{F})$. Pharmacokinetic parameters were evaluated relative to exposure observed in historic studies (18-20 and data on file). Given that many historic studies have had only a single-dose pharmacokinetic sample collection up to 168 hours post-dose, the AUC from time zero to 168 hours postdose $\left(\mathrm{AUC}_{0-168 \mathrm{~h}}\right)$ was also estimated for this study.
Serum concentrations of peginterferon alfa-2a were measured using a validated quantitative sandwich enzymelinked immunosorbent assay method (range 250-4000 pg/mL). The intra-assay precision and accuracy of the quality control samples were $2.5 \%-8.5 \%$ and $-5.5 \%-13.2 \%$, respectively. The interassay precision of the quality control samples were $0.4 \%-9.0 \%$ and $11.7 \%-16.6 \%$, respectively. Serum concentration of anti-peginterferon alfa-2a antibodies was measured by a validated quantitative bridging enzyme-linked immunosorbent assay (assay range 20-1000 ng/mL). The intra-assay precision and accuracy of the quality control samples were $1.3 \%-3.6 \%$ and $-3.2 \%-6.0 \%$, respectively. The interassay precision and accuracy of the quality control samples were $3.9 \%-4.9 \%$ and $4.9 \%-9.0 \%$, respectively. The safety profile and tolerability were assessed throughout the study by monitoring vital signs, laboratory safety tests, electrocardiogram, and adverse events.

\section{Trial 2: user-handling study in patients with chronic hepatitis $C$ \\ Subjects}

Trial 2 enrolled adult patients ( $\geq 18$ years) with chronic hepatitis $\mathrm{C}$ infection who had never previously used an autoinjector. Patients were either treatment-naïve or treatment-experienced (protocol-defined as $\geq 12$ weeks' prior experience with peginterferon). All patients had to be willing and physically able to self-inject the study medication. Females of childbearing potential had a negative urine pregnancy test documented within 24 hours prior to the first dose of study drug and had to be willing to use two forms of contraception during the study. In addition, females of childbearing potential and males with female partners of childbearing potential who were receiving concomitant treatment with ribavirin had to be willing to follow the requirements regarding contraception according to the locally approved label.

Exclusion criteria included the following: hypersensitivity to peginterferon alfa-2a or any of its components; autoimmune hepatitis; a history of, or clinical evidence of, decompensated liver disease; concomitant treatment requiring administration by self-injection; absolute neutrophil count $<1500$ cells $/ \mathrm{mm}^{3}$; platelet count $<90,000$ cells $/ \mathrm{mm}^{3}$; history of severe psychiatric disease; treatment with any investigational drug $\leq 6$ weeks or five half-lives of the drug prior to the first dose of study drug; evidence of serious uncontrolled chronic disease; poorly controlled thyroid dysfunction; evidence of an active or suspected malignancy or a history of malignancy within the previous 2 years where the risk of recurrence was $\geq 20 \%$; history or evidence of severe retinopathy or clinically 
relevant ophthalmologic disorder; and history of major organ transplantation with an existing functional graft.

\section{Study design}

Trial 2 was a two-arm, crossover, open-label, Phase I study ${ }^{20 b}$ conducted in the US in which 60 adult patients with chronic hepatitis $\mathrm{C}$ infection were randomized to $180 \mu \mathrm{g} / 0.5 \mathrm{~mL}$ peginterferon alfa-2a once weekly by either the autoinjector or prefilled syringe for 3 weeks (weeks 1-3) followed by the alternative device (prefilled syringe or autoinjector) for 3 weeks (weeks 4-6). Oral ribavirin (Copegus ${ }^{\circledR}$, Roche, Basel, Switzerland) was administered according to standard of care as per the investigator's judgment. Randomization was centrally controlled; patients were stratified by experience with peginterferon therapy ("yes" vs "no") and randomization numbers were generated by Roche or its designee.

Patients self-administered the subcutaneous injection under observation by a study site staff member. Prior to treatment, a study site staff member demonstrated how to selfadminister using the assigned device by written instructions. The patient was given the written instructions and allowed time to review the instructions and ask questions prior to self-injection.

Patients who required dose modifications or interruptions to manage toxicities associated with peginterferon alfa- $2 \mathrm{a}$ were discontinued from the study because a decreased dose could only be administered using a vial of peginterferon alfa- $2 \mathrm{a}$ and a syringe. The use of supportive therapies to manage toxicities associated with peginterferon and ribavirin was allowed according to local guidelines and standard of care.

\section{Assessments}

The primary objective was to evaluate the successful administration of peginterferon alfa-2a using the autoinjector. Secondary objectives included assessment of tolerability in association with the use of the autoinjector and comparison of the ease of handing an autoinjector versus a prefilled syringe. Data were recorded via an electronic data capture system using electronic case report forms. The safety profile and tolerability were assessed by monitoring vital signs, laboratory safety tests, electrocardiogram, and adverse events. Since there was no validated instrument to evaluate patient-reported outcomes using the disposable autoinjector and prefilled syringe injection devices, assessment of the injection (successful administration and ease of handling) performed by the patient was made upon direct observation by a study site staff member. In addition, a free-text subjective assessment by the patient was collected after each injection.

\section{Injection-method observational survey}

A member of the site study staff observed patient selfadministration of the study drug and evaluated their ability to follow the instructions for each injection method, handle the device, and successfully administer each injection. Observations were recorded based on nine predefined questions, using a "yes" or "no" response (Table 1A).

\section{Patient subjective assessment of injection method}

Prior to completion of the patient subjective assessment of the injection method, the injection-method observational survey was completed to avoid any influence of the patient's assessment on the staff member's observations. The patient subjective assessment consisted of seven predefined questions (Table 1B) and was collected by the study site staff member after each injection.

\section{Statistical analyses}

Both studies were exploratory in nature and therefore no formal statistical comparisons were performed or appropriate. Descriptive analyses describing the results were planned, agreed by health authorities for the user handling study, and performed per protocol. Formal statistical comparisons were not planned and not performed.

Table I Questions for the $(\mathbf{A})$ injection-method observational survey and (B) subjective patient assessment of injection method

A Injection method observational survey*

Did the patient...

I. Exhibit any nervousness prior to the injection?

2. Exhibit any difficulty initiating the injection?

3. Appear confident performing the injection?

4. Follow the instructions for performing the injection without the need for additional instructions or guidance?

5. Experience any technical problems with the device or syringe during the injection?

6. Withdraw the device/syringe before the injection was complete, as evidenced by withdrawal before the second audible click for the autoinjector, and remaining fluid in the barrel for the prefilled syringe?

7. Exhibit any visible pain or physical discomfort (eg, hand discomfort, pain upon injection) during the injection?

8. Appear to be satisfied using the device or syringe?

9. Exhibit any frustration using the syringe or device?

B Patient subjective assessment of injection method

I. Did you have any concerns about using the device before starting the injection?

2. How comfortable were you using the device?

3. Were the instructions easy to follow?

4. Were there any problems with the device itself?

5. Were there any problems performing the injection?

6. Did you experience any pain or discomfort?

7. Is there anything else you would like to add?

Note: *Answers recorded as "yes" or "no". 
In trial 1, peginterferon alfa-2a pharmacokinetic and tolerability data were interpreted in the context of historic peginterferon alfa-2a data from previous clinical studies (8-10 and data on file). A sample size of 50 healthy volunteers was deemed appropriate in support of the autoinjector. Since no statistical comparisons were planned, sample size was not determined by power calculation. All subjects who had received the study medication, whether prematurely withdrawn from the study or not, were included in the safety analysis. Subjects were excluded from the pharmacokinetic analysis population if they significantly violated the inclusion or exclusion criteria, deviated significantly from the protocol, or if data were unavailable or incomplete, which might have influenced the pharmacokinetic analysis.

For trial 2, a sample size of 60 patients was deemed sufficient in support of the autoinjector. As for trial 1, no formal statistical comparisons were planned, and none were performed. Results are summarized using descriptive statistics at scheduled visits. Analyses of data from the injection-method observational survey and patient subjective assessment of injection method survey were performed using an intent-to-treat population, which included all patients who received at least one injection and a survey after injection. The safety analysis population included all patients who received at least one dose of treatment.

\section{Results}

\section{Trial I}

\section{Baseline characteristics}

All 50 healthy subjects, enrolled at one French investigation center (Institut de Pharmacologie Clinique Roche in Strasbourg), completed trial 1 . The majority of subjects were Caucasian, male, of mean age 39 years, and had a mean body mass index of $24.6 \mathrm{~kg} / \mathrm{m}^{2}$ (Table 2). Enrollment began on February 8, 2010 and the study ended on April 16, 2010. Five subjects were not considered evaluable for the pharmacokinetic assessment due to serum levels of peginterferon alfa-2a that were below the limit of quantification of the assay for most, or all, of the samples collected. This phenomenon has been observed in several previous peginterferon alfa-2a pharmacokinetic studies and is considered to be related to endogenous substances present in the serum that interfere with the ability of the enzyme-linked immunosorbent assay to detect peginterferon alfa-2a. Thus, 45 subjects were eligible for pharmacokinetic analysis. All 50 subjects received one dose of study treatment and were included in the safety analysis population.

\section{Pharmacokinetic analysis}

Following a single $180 \mu \mathrm{g}$ dose administered via the disposable autoinjector, mean peginterferon alfa- 2 a serum concentrations peaked at around 48-96 hours postdose. Serum peginterferon alfa- $2 \mathrm{a}$ concentrations were sustained for up to 168 hours (ie, 1 week, Figure 1). The shape of this concentration-time profile suggests sustained absorption and slow elimination, typical of that observed following subcutaneous administration of peginterferon alfa-2a..$^{18,21-23}$ Single-dose pharmacokinetic parameters of peginterferon alfa-2a after administration via the disposable autoinjector are presented in Table 3. Mean peginterferon alfa- $2 \mathrm{a} \mathrm{C}_{\max }$, $\mathrm{AUC}_{0-168 \mathrm{~h}}$, and $\mathrm{T}_{\max }$ were similar to those reported in previous healthy volunteer and patient studies (Figure 2, Table 3) following subcutaneous administration via a vial/syringe or prefilled syringe. Intersubject variability was approximately $30 \%$ for these parameters, and appeared lower than that reported in historic studies (median percent coefficient of variation $[\% \mathrm{CV}] 45 \%$; data on file).

Table 2 Demographic data for trials I and 2

\begin{tabular}{|c|c|c|c|}
\hline & \multirow{2}{*}{$\begin{array}{l}\text { Trial I } \\
\text { (healthy subjects) } \\
\mathbf{n}=\mathbf{5 0}\end{array}$} & \multicolumn{2}{|c|}{ Trial 2 (chronic hepatitis C patients) } \\
\hline & & $\begin{array}{l}\text { Al weeks I-3; PFS weeks 4-6 } \\
n=30\end{array}$ & $\begin{array}{l}\text { PFS weeks I-3; Al weeks 4-6 } \\
n=30\end{array}$ \\
\hline Male, n (\%) & $47(94)$ & $17(57)$ & 14 (47) \\
\hline Mean age, years (range) & $39.0(13.6)$ & $47.6(23-74)$ & $51.3(27-69)$ \\
\hline \multicolumn{4}{|l|}{ Race, n (\%) } \\
\hline White caucasian & $47(94)$ & $26(87)$ & $28(93)$ \\
\hline Black/African & $2(4)$ & $4(13)$ & $2(7)$ \\
\hline Asian & I (2) & 0 & 0 \\
\hline Hispanic/latino ethnicity, n (\%) & $\mathrm{N} / \mathrm{A}$ & $7(23)$ & $4(13)$ \\
\hline Mean body weight, kg (SD) & $77.1(9.9)$ & $88.1(23.2)$ & $83.4(17.4)$ \\
\hline Mean BMI, kg/m² (SD) & $24.6(2.7)$ & $31.0(8.4)$ & $28.9(5.2)$ \\
\hline
\end{tabular}

Note: For trial 2, patient demographic data are presented according to which device they were randomized to first. Abbreviations: BMI, body mass index; N/A, not assessed; SD, standard deviation; Al, autoinjector; PFS, prefilled syringe. 

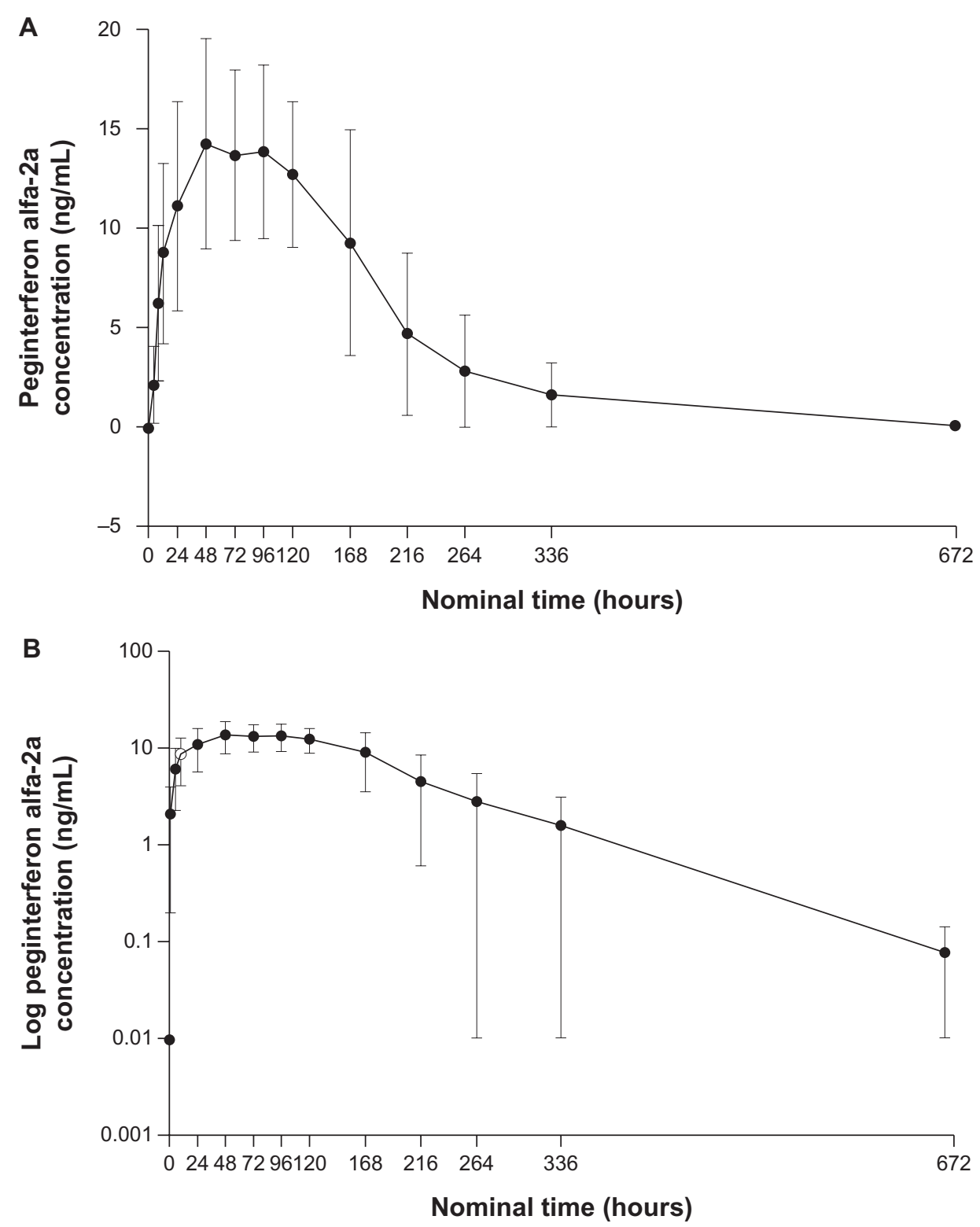

Figure I Mean ( \pm standard deviation) peginterferon alfa-2a concentration-time profile after single dose administered via the disposable autoinjector on linear (A) and log (B) scales.

\section{Tolerability}

Forty-three of 50 subjects ( $86 \%$ ) reported at least one adverse event; the 43 subjects experienced a total of 81 adverse events. The most common adverse events reported were influenza-like illness (40\%), headache (34\%), myalgia (24\%), injection site erythema (12\%), and pyrexia $(10 \%)$; most adverse events were mild or moderate in intensity (80/81 events) and were judged by the investigator as being related to study medication (75/81). No serious adverse events or deaths occurred during the trial, and no significant electrocardiographic abnormalities were reported. No subject withdrew prematurely due to an adverse event or laboratory abnormalities.
Thirty subjects had a total of 109 laboratory abnormalities, the majority of which were decreased neutrophils and white blood cell count ( $n=25$ and $n=15$, respectively); none were considered clinically significant by the investigator. Two subjects $(4 \%)$ tested positive for binding antibodies to peginterferon alfa-2a at follow-up after administration of peginterferon alfa-2a via the disposable autoinjector, similar to that described in the package insert for peginterferon alfa-2a $(9 \%-29 \%) .{ }^{24}$

\section{Trial 2}

\section{Baseline characteristics}

In trial 2, 60 patients were enrolled at ten centers in the United States (treatment-experienced, $n=29$; treatment-naïve, $n=31$ ). 
Table 3 Single-dose pharmacokinetic parameters of peginterferon alfa-2a after administration via disposable autoinjector compared with historic studies

\begin{tabular}{|c|c|c|c|c|c|c|c|}
\hline \multirow[t]{3}{*}{ Parameter (units) } & \multirow[t]{3}{*}{$\mathbf{N}$} & \multirow[t]{3}{*}{ Mean \pm SD } & \multirow[t]{3}{*}{$\% C V$} & \multicolumn{4}{|c|}{ Historic studies } \\
\hline & & & & \multicolumn{2}{|c|}{ Healthy volunteer studies* } & \multicolumn{2}{|c|}{ Hepatitis C patient studies ${ }^{\dagger}$} \\
\hline & & & & Mean, range & $\% C V$, range & Mean, range & $\% \mathrm{CV}$, range \\
\hline $\mathrm{T}_{\max }$ (hours) & 45 & $78.7 \pm 43.7$ & 55.5 & $78.0-102$ & $28.0-48.0$ & $57.0-105.0$ & $35.0-70.2$ \\
\hline $\mathrm{C}_{\max }(\mathrm{ng} / \mathrm{mL})$ & 45 & $16.1 \pm 5.3$ & 33.1 & $9.0-14.2$ & $17.6-38.8$ & $8.0-15.4$ & $26.7-62.4$ \\
\hline $\mathrm{AUC}_{0-168 \mathrm{~h}}(\mathrm{ng} \cdot \mathrm{hour} / \mathrm{mL})$ & 45 & $1996 \pm 613$ & 30.7 & $950-1743$ & $26.3-50.2$ & $1009-1820$ & $32.2-79.3$ \\
\hline $\mathrm{AUC}_{0 \text {-last }}(\mathrm{ng} \cdot \mathrm{hour} / \mathrm{mL})$ & 45 & $2736 \pm 1054$ & 38.5 & & & & \\
\hline $\mathrm{AUC}_{0-\infty}(\mathrm{ng} \cdot \mathrm{hour} / \mathrm{mL})$ & 43 & $3048 \pm 1062$ & 34.9 & & & & \\
\hline $\mathrm{t}_{1 / 2}$ (hours) & 43 & $67.8 \pm 36.3$ & 53.5 & & & & \\
\hline $\mathrm{V}_{z} / \mathrm{F}(\mathrm{L})$ & 43 & $5.9 \pm 2.9$ & 49.5 & & & & \\
\hline $\mathrm{Cl} / \mathrm{F}$ (mL/hour) & 43 & $67.5 \pm 27.1$ & 40.1 & & & & \\
\hline
\end{tabular}

Notes: *NPI5537, NPI5762, NPI5989, NPI5580, NPI5538; `NVI5489, NVI5495, NVI5496, NVI5497, NPI7354, and NPI7355.

Abbreviations: $A \cup C$, area under the concentration-time curve; $C_{\max }$, observed maximum serum concentration; $\mathrm{Cl} / \mathrm{F}$, apparent total body clearance; $T_{\max }$, time to $C_{\max }$; $t_{1 / 2}$, terminal elimination half-life; $V_{z} / F$, apparent volume of distribution; \%CV, percent coefficient of variation; $\mathrm{Al}$, autoinjector; PFS, prefilled syringe.

Enrollment began in March 2010 and the study ended in May 2010. Fifty-seven patients completed the study. The three withdrawals occurred during the autoinjector period and were due to adverse events or laboratory abnormalities. All 60 patients received treatment via the autoinjector and 59 received treatment via the prefilled syringe; one patient, who received the autoinjector first, discontinued during this phase and thus did not receive the prefilled syringe. Therefore, the safety analysis includes 60 patients for the autoinjector period and 59 for the prefilled syringe period. Survey data from all 60 patients are included in the survey analyses.

The majority of patients were Caucasian, with a mean age of 49 years and a mean body mass index of $30 \mathrm{~kg} / \mathrm{m}^{2}$. Overall, there were no major differences between patients randomized to the disposable autoinjector first, followed by prefilled syringe and patients randomized to the prefilled syringe first followed by autoinjector (Table 2).
Baseline characteristics were also similar between treatmentnaïve and treatment-experienced patients (data not shown). Almost all patients were receiving concomitant medications at the start of the study as was expected for a hepatitis C-infected population.

\section{Injection-method observational survey results}

Results of the injection-method observational survey are summarized for each injection $(1,2$, or 3$)$ by injection method used (disposable autoinjector or prefilled syringe), regardless of timing (weeks 1-3 vs weeks 4-6, Table 4). Overall, few patients were observed to have handling difficulties with either device. For both devices, more user-handling issues tended to be reported by the observer during the first injection than during the second or third injection; this included issues related to the user's mental state, such as nervousness

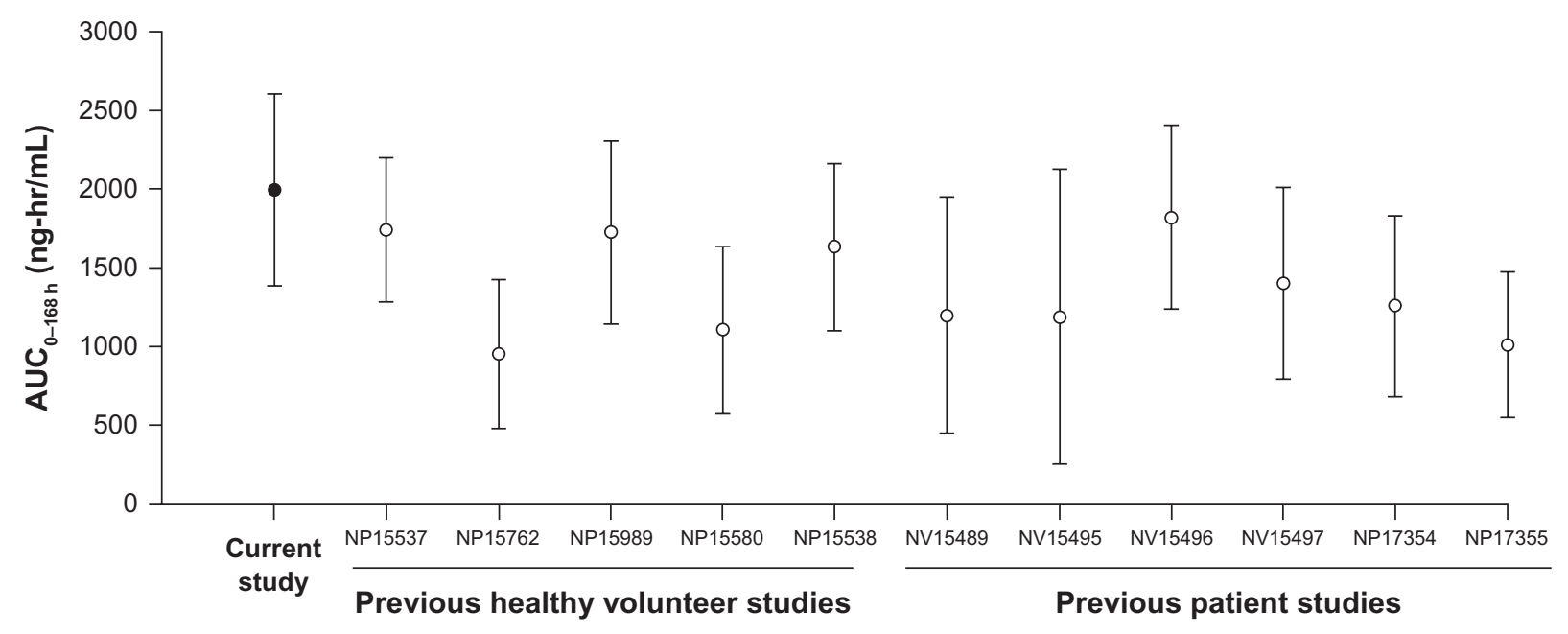

Figure 2 Mean ( \pm standard deviation) exposure from previous peginterferon alfa-2a healthy volunteer and patient studies. 
Table 4 Summary of injection-method observational survey responses by study staff

\begin{tabular}{|c|c|c|c|c|c|c|}
\hline \multirow[t]{2}{*}{ n (\%) } & \multicolumn{2}{|c|}{ Injection I } & \multicolumn{2}{|c|}{ Injection 2} & \multicolumn{2}{|c|}{ Injection 3} \\
\hline & $\begin{array}{l}\text { Al } \\
n=60\end{array}$ & $\begin{array}{l}\text { PFS } \\
n=59\end{array}$ & $\begin{array}{l}\text { Al } \\
n=58\end{array}$ & $\begin{array}{l}\text { PFS } \\
n=59\end{array}$ & $\begin{array}{l}\text { Al } \\
n=57\end{array}$ & $\begin{array}{l}\text { PFS } \\
n=59\end{array}$ \\
\hline Patients exhibited nervousness prior to injection & II (I8) & $14(24)$ & 0 & $3(5)$ & $\mathrm{I}(2)$ & $2(3)$ \\
\hline Patients exhibited difficulty initiating injection & $7(12)$ & $10(17)$ & 0 & $2(3)$ & 0 & $2(3)$ \\
\hline Patients appeared confident performing the injection & $58(97)$ & $46(78)$ & $58(100)$ & $57(97)$ & $57(100)$ & $58(98)$ \\
\hline Patients successfully followed instructions & $58(97)$ & $54(92)$ & $53(91)$ & $53(90)$ & $55(96)$ & $56(95)$ \\
\hline Patients experienced technical difficulties with device & $7(12)$ & $2(3)$ & $5(9)$ & $I(2)$ & $3(5)$ & $3(5)$ \\
\hline Patients withdrew device before completion of injection & $4(7)$ & 0 & $\mathrm{I}(2)$ & 0 & 0 & I (2) \\
\hline Patients exhibited pain/discomfort on injection & 0 & $2(3)$ & 0 & I (2) & 0 & $2(3)$ \\
\hline Patients appeared satisfied using device & $60(100)$ & $51(86)$ & $58(100)$ & $55(93)$ & $57(100)$ & $56(95)$ \\
\hline Patients exhibited frustration with device & I (2) & $2(3)$ & 0 & $2(3)$ & 0 & $2(3)$ \\
\hline
\end{tabular}

Notes: Results summarized for each injection (I, 2, or 3) by injection method used (disposable Al or PFS), regardless of timing (weeks I-3 vs weeks 4-6).

Abbreviations: Al, autoinjector; PFS, prefilled syringe.

and lack of confidence, as well as issues related to operating the device, such as difficulty initiating injection, withdrawal before injection was complete, and difficulties in using the device correctly.

On first injection, slightly more patients using the autoinjector had technical difficulties with the device $(12 \%$; prefilled syringe $3 \%$ ) and withdrew before the injection was complete (7\%; prefilled syringe $0 \%$ ). However, user handling tended to improve during the second and third injections for both devices, and by the third injection, reported difficulties were low for both devices.

For the prefilled syringe device, a few patients $(2 \%-10 \%)$ were identified to have issues with most of the nine criteria in the observational survey during the second and third injections. For the disposable autoinjector device, a few patients were observed to have technical problems (5\%-9\%) and difficulty following instructions (4\%-9\%) during the second and third injections. However, patients were generally observed to be more satisfied, able to follow instructions better, more confident, and more able to initiate injection successfully with the autoinjector device compared with the prefilled syringe device (Table 4). Following first injection, all patients (100\%) using the autoinjector device and $93 \%-98 \%$ of patients using the prefilled syringe successfully initiated injection and were confident and satisfied with the respective device. Pain/discomfort was not observed in any patients using the autoinjector, but a few patients $(2 \%-3 \%)$ were observed to experience pain/discomfort with the prefilled syringe for each of the three injections.

In general, treatment-experienced patients were observed to have less difficulty with the first injection than treatment-naive patients, particularly when using the prefilled syringe (difficulty initiating injection: autoinjector $14 \%$ vs $10 \%$; prefilled syringe $4 \%$ vs $29 \%$; and technical problems $10 \%$ vs $13 \%$; prefilled syringe $0 \%$ vs $6 \%$, respectively). Treatment-experienced patients were also observed to be less nervous than treatment-naïve patients prior to first injection (autoinjector 9\% vs 29\%; prefilled syringe $7 \%$ vs 39\%). However, there were no differences evident between the two patient groups on subsequent injections.

\section{Patient subjective assessment}

The patient subjective assessment also reflected a positive patient opinion on the autoinjector device, including a high comfort level and lack of pain compared with the prefilled syringe device (Table 5). All patients reported that the instructions were easy to follow for both devices. Twenty-five percent of patients reported concern about using the autoinjector prior to the first injection (25\%) compared with $17 \%$ using the prefilled syringe. Patient concern was generally reported to be due to apprehension and not knowing what to expect with a new device; no patients reported concern on subsequent injections with the autoinjector, and all patients were comfortable using the device.

A few patients (approximately 5/60) reported problems with the autoinjector device or performing the injection; these included failure to release the thumb immediately upon injection and removal of the device before injection was complete. Several comments were related to the second click that indicates delivery of the full dose, with one patient failing to observe a second click and two patients removing the device prematurely. These comments reflect the technical difficulties, lack of adherence to instructions, and withdrawal before completion reported in the observational survey for the three injections (Table 4). 
Table 5 Summary of patient subjective assessment of the injection method*

\begin{tabular}{|c|c|c|c|c|c|c|}
\hline \multirow[t]{2}{*}{ The patient } & \multicolumn{2}{|c|}{ Injection I } & \multicolumn{2}{|c|}{ Injection 2} & \multicolumn{2}{|c|}{ Injection 3} \\
\hline & $\begin{array}{l}\text { Al } \\
n=60\end{array}$ & $\begin{array}{l}\text { PFS } \\
n=59\end{array}$ & $\begin{array}{l}\text { Al } \\
n=59\end{array}$ & $\begin{array}{l}\text { PFS } \\
\mathrm{n}=\mathbf{5 9}\end{array}$ & $\begin{array}{l}\text { Al } \\
n=57\end{array}$ & $\begin{array}{l}\text { PFS } \\
n=59\end{array}$ \\
\hline Was concerned about using the device preinjection & $15(25)$ & $10(17)$ & 0 & $3(5)$ & 0 & $2(3)$ \\
\hline Was comfortable using the device & $56(93)$ & $52(88)$ & $58(100)$ & $55(93)$ & $57(100)$ & $58(98)$ \\
\hline Found instructions easy to follow & $60(100)$ & $59(100)$ & $58(100)$ & $59(100)$ & $57(100)$ & $59(100)$ \\
\hline Reported problems with the device & $4(7)$ & 0 & I (2) & $2(3)$ & 0 & $2(3)$ \\
\hline Reported problems performing injection & $3(5)$ & $\mathrm{I}(2)$ & $4(7)$ & 0 & I (2) & 0 \\
\hline Reported pain or discomfort & $2(3)$ & $8(14)$ & 0 & $3(5)$ & 0 & $4(7)$ \\
\hline
\end{tabular}

Note: *Results summarized for each injection (I, 2, or 3) by injection method used (disposable AI or PFS), regardless of timing (weeks I-3 vs weeks 4-6).

Abbreviations: Al, autoinjector; PFS, prefilled syringe.

For the final part of the survey, patients were asked to provide any further comments. Nearly half of all patients indicated a preference for the autoinjector device over the prefilled syringe ( $n=26 ; 43 \%)$. Reasons for preferring the autoinjector included: it was easier, quicker, and safer to use; less painful; less bruising at injection site; less preparation time; no view of the needle which reduced anxiety; cannot be reused; less of a reminder of how the virus was transmitted. Two patients $(3 \%)$ reported that they felt more confident using the prefilled syringe device because by being able to see the medication in the syringe they were more certain that they had successfully administered the drug. The remaining patients $(n=32 ; 53 \%)$ either did not offer further comment or did not state a preference of one device over the other.

\section{Tolerability}

Overall, both devices were well tolerated, with similar adverse events reported, consistent with the known safety profile of peginterferon alfa-2a plus ribavirin. The most common adverse events $(>10 \%)$ reported during both the autoinjector and prefilled syringe periods were nausea $(13 \%$ and $14 \%$, respectively) and fatigue (13\% and $12 \%$, respectively). No serious adverse events or deaths occurred during this trial. Three patients were withdrawn from the study for adverse events of pulmonary sarcoidosis and thrombocytopenia and a laboratory abnormality of low neutrophil count (due to the need of dose modification, as per the protocol).

A slightly higher percentage of patients reported an adverse event during the prefilled syringe period (64\%) versus the autoinjector period (57\%) but this difference of four patients was not considered to be clinically relevant. The majority of adverse events were of mild or moderate intensity ( $96 \%$ and $99 \%$ events during the autoinjector and prefilled syringe periods, respectively) and were judged by the investigator to be related to the study medication
(94\% and 98\% events during the autoinjector and prefilled syringe periods, respectively).

The incidence of individual reported adverse events was similar in the autoinjector and prefilled syringe periods with the exception of diarrhea ( $7 \%$ and $14 \%$, respectively), arthralgia ( $10 \%$ and $5 \%$, respectively), and insomnia ( $8 \%$ and $3 \%$, respectively). However, given the relatively small numbers of patients reporting these events, the difference between the autoinjector and prefilled syringe was not considered clinically different. Very few injection site reactions were reported (six patients during the autoinjector period and three patients during the prefilled syringe period).

In general, treatment-experienced patients reported adverse events less frequently (55\% and $43 \%$ for the autoinjector and prefilled syringe periods, respectively) than treatment-naïve patients ( $58 \%$ and $84 \%$, respectively). In treatment-naïve patients, adverse event reporting was more frequent during the prefilled syringe period (84\%) than during the autoinjector period (58\%), and the most common adverse events were general disorders (eg, fatigue, pain, chills, and irritability) and injection site reactions. For the treatmentnaive patients and treatment-experienced patients, there were no clinically relevant differences between the devices in the adverse events reported.

The most common marked laboratory abnormality was neutropenia during both the autoinjector and prefilled syringe periods ( $34 \%$ and $33 \%$, respectively). Other reported marked laboratory abnormalities during both the autoinjector and prefilled syringe periods included low white blood cell count (19\% and $16 \%$, respectively), low platelets ( $9 \%$ and $5 \%$, respectively), low lymphocyte count ( $9 \%$ and $2 \%$, respectively), and low hemoglobin ( $5 \%$ and $5 \%$, respectively). The majority of the significant laboratory abnormalities were reported in treatment-naïve patients (treatment-naïve vs treatment-experienced: neutropenia $39 \%-45 \%$ vs $22 \%-26 \%$; 
low white cell count $23 \%-26 \%$ vs $7 \%-11 \%$; and low platelets $10 \%-13 \%$ vs $0 \%-4 \%$ ).

No study-onset electrocardiogram abnormalities were reported, and there was no apparent pattern of clinically significant changes in vital signs during this study.

\section{Discussion}

Together, the two registration studies presented here evaluated a new disposable autoinjector that has undergone development for the administration of peginterferon alfa-2a. The studies assessed the pharmacokinetics, safety profile, tolerability, and ease of administration of peginterferon alfa-2a by healthy subjects and patients with chronic hepatitis $\mathrm{C}$. Overall, the findings indicate that peginterferon alfa- 2 a can be successfully delivered via this device, and are comparable with that using the approved prefilled syringe. In addition, patients perceived the autoinjector as easier and more convenient to use, and tended to show preference over the prefilled syringe.

Importantly, data from the 45 evaluable healthy subjects in the pharmacokinetic study demonstrate that peginterferon alfa-2a, when administered via the disposable autoinjector, has a mean exposure similar to that previously reported using a vial and syringe or a prefilled syringe (data on file). The mean $\mathrm{AUC}_{0-168 \mathrm{~h}}$ in the present study was slightly higher relative to previous studies, but drug exposure was within the range of previous studies and the difference was not considered to be clinically relevant. Although speculative, the difference in intersubject variability observed may be due to greater inconsistencies in administration techniques associated with manual injection, where the user has to control the depth, speed, and completeness of injection. In contrast, the disposable autoinjector is fully automated, and thus variability in injection technique may be decreased.

Data from this present analysis also indicate that patients can successfully administer peginterferon alfa-2a using the new autoinjector device. Survey results indicate that instructions for use were easy for patients to follow and that there was no evidence of pain or discomfort observed during the use of the autoinjector device. In addition, patients generally perceived the autoinjector to be more convenient, easier, quicker, and simpler to use, and were more satisfied with its use than the prefilled syringe. These findings are concordant with other similar sized studies that have compared patient satisfaction and preference between the autoinjector and prefilled syringe devices. ${ }^{11-13}$ However, two patients did express a preference for the prefilled syringe device, with the primary reason being that they were more confident that they had successfully injected the drug. This finding was used to support revision of the patient instructions and training programs for use of the autoinjector, to ensure patients are trained to know when they have successfully completed their injection of the drug.

One obvious advantage with the autoinjector device is that the needle is shielded from view and the user does not need to manually insert the needle into the skin. This is an important benefit for patients with a fear of needles as well as for previous intravenous drug users who often express fear that treatment for hepatitis $\mathrm{C}$ virus will cause them to relapse back into substance abuse. ${ }^{25,26}$ The possession of a syringe used for the interferon injections and/or the action of drawing up the interferon for the injection may stimulate a patient's desire for drugs. ${ }^{25,26}$ Therefore, by eliminating both of these factors, the autoinjector has the potential to reduce the risk of recidivism in substance abusers. This is important given that the prevalence rate of hepatitis $\mathrm{C}$ infection among injection drug users ranges between $40 \%$ and $90 \%$, depending on geographic location and duration of exposure to injection drug use. ${ }^{27}$

Poor adherence to treatment is a universal issue in the management of all chronic conditions and can have serious consequences, including reduced efficacy and increased health care costs. ${ }^{28-30}$ In hepatitis C, optimal adherence to therapy is critical for a successful treatment outcome. ${ }^{31}$ Among patients with hepatitis $\mathrm{C}$ genotype 1 infection, sustained virologic response rates are significantly greater in those who receive at least $80 \%$ of the total peginterferon and ribavirin dose for more than $80 \%$ of the scheduled treatment duration compared with patients who fail to achieve these adherence criteria (51\% vs 34\%; $P=0.011) .{ }^{32}$ Hughes and Shafran ${ }^{33}$ confirmed that treatment response is not only influenced by hepatitis $C$ genotype and viral load, but also by patient-related factors, including adherence. Thus, improving adherence is an important goal in the management of hepatitis $\mathrm{C}$. By improving convenience and shielding the needle from view, findings from this study suggest the autoinjector may have the potential to help patients overcome injection-related problems, such as pain/discomfort, anxiety, fear of needles, fear of recidivism, and worry over incorrect injection techniques, all of which may improve patient adherence to study medication, which can subsequently increase the potential to achieve viral suppression.

The use of the autoinjector did not raise any new safety concerns, and the adverse events reported were consistent 
with the known safety profile of peginterferon alfa-2a among patients with hepatitis C. ${ }^{34-36}$ The autoinjector itself also offers administrational safety features, the primary one being lack of needle exposure. This reduces the risk of needlestick injury, which is a key benefit for the introduction of autoinjector devices. Needlestick injury is an important issue that needs to be addressed, given that $2 \%-4 \%$ of acute hepatitis $\mathrm{C}$ infections that occur annually are reported among health care workers. ${ }^{8,37}$ In the US alone, 69,000 injuries a year could be prevented through the use of safer devices. ${ }^{38}$ Furthermore, with the autoinjector, the needle cannot be reused, which was viewed as an advantage by some patients because many chronic hepatitis $\mathrm{C}$ patients are intravenous drug users or former users.

The positive findings of these analyses should be considered in the context of the study limitations. With the pharmacokinetic study, there was no control group and thus data were compared with the historic studies presented in Figure 2. In addition, while tolerability data from this pharmacokinetic study were consistent with those reported previously with peginterferon alfa-2a in clinical trials, ${ }^{24,35,36}$ it must be recognized that this was a single-dose study and cannot be directly compared with the safety profile of a $24-48$-week treatment with peginterferon alfa-2a.

It was not possible to blind study participants in the user handling and tolerability study. It is possible that, for treatment-experienced patients who were already satisfied and comfortable with their existing administration method, changing their injection practice may have resulted in lower satisfaction with the autoinjector, particularly considering the short duration of the study treatment. In addition, treatmentnaïve patients or patients who have previously discontinued treatment due to injection-related issues may have perceived a greater benefit in using a new device. Assessment of the attitudes of health care practitioners was not included in the study; evaluation of their use and preferences would be of clinical interest, particularly in terms of ease of training patients and patient compliance.

However, despite these limitations, the findings from this study provide sufficient evidence to support the use of the autoinjector as a delivery option for patients.

Although the management of chronic hepatitis $\mathrm{C}$ is entering a new era with the recent approval of the protease inhibitors, telaprevir and boceprevir, ${ }^{39-42}$ these agents are approved only in combination with peginterferon alfa plus ribavirin. ${ }^{43,44}$ Therefore, delivery devices for peginterferon alfa-2a, particularly those that can help simplify treatment strategies such as the autoinjector, will continue to be of clinical relevance for the foreseeable future.

\section{Conclusion}

The results of the analyses reported here indicate that peginterferon alfa-2a can be successfully and safely delivered using the disposable autoinjector, and the serum exposure of a single dose of peginterferon alfa- $2 \mathrm{a}$ is similar to that observed previously using a vial and syringe or prefilled syringe. Importantly, patients reported that the instructions were easy to follow, the device was easy to handle, and there were no reported issues in tolerability in comparison with the prefilled syringe.

\section{Acknowledgments}

This analysis was funded by F Hoffman-La Roche Ltd. Support for third-party writing assistance for this manuscript was provided by F Hoffmann-La Roche Ltd. The authors would like to thank Priya Sriraman and Gennady Samokhin for their bioanalytical expertise and support of the pharmacokinetic study.

\section{Disclosure}

PV and GM have no disclosures to make. EL has received research/grant support from: Abbott Laboratories, Achillion Pharmaceuticals, Anadys Pharmaceuticals, Biolex Therapeutics, Boehringer Ingelheim, Bristol-Myers Squibb, Gilead Sciences, GlaxoSmithKline, GlobeImmune, Idenix Pharmaceuticals, Idera Pharmaceuticals, Inhibitex Pharmaceuticals, Medarex, Medtronic, Merck \& Co, Novartis, Pharmasset, Roche, Sanofi-Aventis, Schering-Plough, Santaris Pharmaceuticals, Scynexis Pharmaceuticals, Tibotec, Vertex Pharmaceuticals, ViroChem Pharma, and ZymoGenetics. EL has been an advisory board participant for Abbott Laboratories, Achillion Pharmaceuticals, Anadys Pharmaceuticals, Biolex Therapeutics, GlobeImmune, Inhibitex Pharmaceuticals, Merck \& Co, and Pharmasset, and has been a speaker for Gilead, Merck, and Vertex. KLB is on the Genentech Speakers Bureau. AB, $\mathrm{BB}, \mathrm{CB}, \mathrm{CW}$, JS, NL, and RL are employees of Roche.

\section{References}

1. Ghany MG, Strader DB, Thomas DL, Seeff LB. Diagnosis, management, and treatment of hepatitis C: an update. Hepatology. 2009;49(4):1335-1374.

2. Lok AS, McMahon BJ. Chronic hepatitis B: update 2009. Hepatology. 2009;50(3):661-662

3. Zeuzem S, Welsch C, Herrmann E. Pharmacokinetics of peginterferons. Semin Liver Dis. 2003;23 Suppl 1:23-28.

4. Pedder SC. Pegylation of interferon alfa: structural and pharmacokinetic properties. Semin Liver Dis. 2003;23 Suppl 1:19-22. 
5. Pegasys. Summary of product characteristics. Available from: http:// www.ema.europa.eu/docs/en_GB/document_library/EPAR_-_ Summary_for_the_public/human/000395/WC500039196.pdf. Accessed September 20, 2011.

6. Schering Corporation. PegIntron ${ }^{\circledR}$ (peginterferon alfa-2b) prescribing information. http://www.spfiles.com/pipeg-intron.pdf. Accessed October 7, 2011.

7. Hoffmann-La Roche Inc. Medication Guide. Pegasys ${ }^{\circledR}$. Solution for subcutaneous injection. Available from: http://www.gene.com/gene/ products/information/pegasys/pdf/Pegasys_MedGuide.pdf. Accessed October 7, 2011.

8. National Institute for Occupational Safety and Health. Preventing needlestick injuries in health care settings. Available from: http://www. cdc.gov/niosh/docs/2000-108/. Accessed September 22, 2011.

9. Cox D, Stone J. Managing self-injection difficulties in patients with relapsing-remitting multiple sclerosis. J Neurosci Nurs. 2006; 38(3):167-171.

10. Devonshire V, Arbizu T, Borre B, et al. Patient-rated suitability of a novel electronic device for self-injection of subcutaneous interferon beta-1a in relapsing multiple sclerosis: an international, single-arm, multicentre, Phase IIIb study. BMC Neurol. 2010;10(1):28.

11. Kivitz A, Cohen S, Dowd JE, et al. Clinical assessment of pain, tolerability, and preference of an autoinjection pen versus a prefilled syringe for patient self-administration of the fully human, monoclonal antibody adalimumab: the TOUCH trial. Clin Ther. 2006;28(10):1619-1629.

12. Paul C, Stalder J, Thaci D, et al. Patient satisfaction with injection devices: a randomized controlled study comparing two different etanercept delivery systems in moderate to severe psoriasis. $J$ Eur Acad Dermatol Venereol. 2011. [Epub ahead of print]. doi: 10.1111/j.14683083.2011.04093.x

13. Berteau C, Schwarzenbach F, Donazzolo Y, et al. Evaluation of performance, safety, subject acceptance, and compliance of a disposable autoinjector for subcutaneous injections in healthy volunteers. Patient Prefer Adherence. 2010;4:379-388.

14. Borras-Blasco J, Gracia-Perez A, Rosique-Robles JD, Castera MD, Abad FJ. Acceptability of switching adalimumab from a prefilled syringe to an autoinjection pen. Expert Opin Biol Ther. 2010;10(3):301-307.

15. Graff MR, McClanahan MA. Assessment by patients with diabetes mellitus of two insulin pen delivery systems versus a vial and syringe. Clin Ther. 1998;20(3):486-496.

16. Lee WC, Balu S, Cobden D, Joshi AV, Pashos CL. Medication adherence and the associated health-economic impact among patients with type 2 diabetes mellitus converting to insulin pen therapy: an analysis of thirdparty managed care claims data. Clin Ther. 2006;28(10):1712-1725.

17. Rubin RR, Peyrot M. Quality of life, treatment satisfaction, and treatment preference associated with use of a pen device delivering a premixed 70/30 insulin aspart suspension (aspart protamine suspension/ soluble aspart) versus alternative treatment strategies. Diabetes Care. 2004;27(10):2495-2497.

18. Algranati N, Sy S, Modi M, et al. A branchedmethoxy $40 \mathrm{kDa}$ polyethylene glycol (PEG) moiety optimizes the pharmacokinetics (PK) of peginterferon $\alpha-2 \mathrm{~A}$ and may explain its enhanced efficacy in chronic hepatitis C (CHC) [abstract]. Hepatology. 1999;30 Suppl Pt 2:190.

19. Matin M, Modi M. Characterization of pegylated ( $40 \mathrm{kDa}$ ) interferon alfa-2a (Pegasys) in the elderly [abstract]. Hepatology. 2000;32 Suppl Pt 2:348.

20. Modi M, Fried M, Reindollar R, et al. The pharmacokinetic behavior of pegylated (40 kDa) interferon alfa-2a (Pegasys) in chronic hepatitis C patients after multiple dosing [Abstract]. Hepatology. 2000;32 Suppl Pt 2:394.

20b. Hoffmann-La Roche. A Study of Administration of Peginterferon Alfa-2a [Pegasys] by Autoinjector Versus Pre-filled Syringe in Patients With Chronic Hepatitis C. In: clinicaltrials.gov [Internet]. Bethesda, MD: National Library of Medicine (US). March 9, 2010 [last updated on June 15, 2011]. Available from: http://clinicaltrials.gov/ct2/show/ NCT01087944. NLM identifier: NCT01087944.
21. Nieforth KA, Nadeau R, Patel IH, Mould D. Use of an indirect pharmacodynamic stimulation model of MX protein induction to compare in vivo activity of interferon alfa- $2 \mathrm{a}$ and a polyethylene glycol-modified derivative in healthy subjects. Clin Pharmacol Ther. 1996;59(6):636-646.

22. Zeuzem S, Feinman SV, Rasenack J, et al. Peginterferon alfa-2a in patients with chronic hepatitis C. N Engl J Med. 2000;343(23): $1666-1672$.

23. Xu Z-X, Hoffman J, Patel I, Joubert P. Single-dose safety/tolerability and pharmacokinetic/pharmacodynamics (PK/PD) following administration of ascending subcutaneous doses of pegylated-interferon (PEG-IFN) and interferon $a-2 \mathrm{a}$ (IFN $a-2 \mathrm{a}$ ) to healthy subjects [Abstract]. Hepatology. 1998;28:702A.

24. Genetech USA I. Pegasys full prescribing information and medication guide. Available from: http://www.gene.com/gene/products/information/ pegasys/pdf/pi.pdf. Accessed October 7, 2011.

25. Ogawa L. Substance use experiences and hepatitis c treatment decisionmaking among HIV/HCV co-infected adults: A dissertation. 2007. University of Massachusetts Medical School. GSN Dissertations. Paper 3.

26. Bova C, Ogawa L, Sullivan-Bolyai S. Hepatitis C treatment experiences and decision-making among patients living with HIV infection. JAssoc Nurses AIDS Care. 2010;21:63-74.

27. Hagan H, Pouget ER, Des J. A systematic review and meta-analysis of interventions to prevent hepatitis $\mathrm{C}$ virus infection in people who inject drugs. J Infect Dis. 2011;204(1):74-83.

28. Osterberg L, Blaschke T. Adherence to medication. $N$ Engl J Med. 2005;353(5):487-497.

29. Simpson SH, Eurich DT, Majumdar SR, et al. A meta-analysis of the association between adherence to drug therapy and mortality. $B M J$. 2006;333(7557): 15 .

30. [No authors listed]. Poor adherence to long-term treatment of chronic diseases is a worldwide problem. Rev Panam Salud Publica. 2003;14(3):218-221.

31. Cacoub P, Ouzan D, Melin P, et al. Patient education improves adherence to peg-interferon and ribavirin in chronic genotype 2 or 3 hepatitis C virus infection: a prospective, real-life, observational study. World $J$ Gastroenterol. 2008;14(40):6195-6203.

32. McHutchison JG, Manns M, Patel K, et al. Adherence to combination therapy enhances sustained response in genotype-1-infected patients with chronic hepatitis C. Gastroenterology. 2002;123(4):1061-1069.

33. Hughes CA, Shafran SD. Chronic hepatitis C virus management: 2000-2005 update. Ann Pharmacother. 2006;40(1):74-82.

34. Fontanges T, Beorchia S, Douvin C, et al. Safety and efficacy of combination therapy with peginterferon alfa-2a (40kD) and ribavirin in the outpatient setting: prospective analysis of 197 patients with chronic hepatitis C viral infection. Gastroenterol Clin Biol. 2007;31(6-7):566-572.

35. Fried MW, Shiffman ML, Reddy KR, et al. Peginterferon alfa-2a plus ribavirin for chronic hepatitis C virus infection. $N$ Engl J Med. 2002;347(13):975-982.

36. Hadziyannis SJ, Sette H Jr, Morgan TR, et al. Peginterferon-alpha2a and ribavirin combination therapy in chronic hepatitis $\mathrm{C}$ : a randomized study of treatment duration and ribavirin dose. Ann Intern Med. 2004;140(5):346-355.

37. Alter MJ. Epidemiology of hepatitis C. Hepatology. 1997;26 Suppl 1: $62 \mathrm{~S}-65 \mathrm{~S}$.

38. United States General Accounting Office. Occupational safety: selected cost and benefit implications of needlestick prevention devices for hospitals. Document GAO-01-60R. Washington, DC. 2000.

39. Jacobson IM, McHutchison JG, Dusheiko G, et al. Telaprevir for previously untreated chronic hepatitis $\mathrm{C}$ virus infection. $N$ Engl $J$ Med. 2011;364(25):2405-2416.

40. Zeuzem S, Andreone P, Pol S, et al. Telaprevir for retreatment of HCV infection. N Engl J Med. 2011;364(25):2417-2428.

41. Poordad F, McCone J Jr, Bacon BR, et al. Boceprevir for untreated chronic HCV genotype 1 infection. N Engl J Med. 2011;364(13): 1195-1206. 
42. Bacon BR, Gordon SC, Lawitz E, et al. Boceprevir for previously treated chronic HCV genotype 1 infection. $N$ Engl $J$ Med. 2011;364(13):1207-1217.

43. Merck \& Co. I. Victrelis (boceprevir) prescribing information. Available from: http://www.accessdata.fda.gov/drugsatfda_docs label/2011/2022581bl.pdf. Accessed October 7, 2011.
44. Vertex Pharmaceuticals Ltd. Incivek (telaprevir) prescribing information. Available from: http://www.natap.org/2011/HCV/INCIVEKUSPI. pdf. Accessed October 7, 2011.

\section{Publish your work in this journal}

Patient Preference and Adherence is an international, peer-reviewed, open access journal focusing on the growing importance of patient preference and adherence throughout the therapeutic continuum. Patient satisfaction, acceptability, quality of life, compliance, persistence and their role in developing new therapeutic modalities and compounds to optimize clinical outcomes for existing disease states are major areas of interest. This journal has been accepted for indexing on PubMed Central. The manuscript management system is completely online and includes a very quick and fair peer-review system. Visit http://www.dovepress.com/ testimonials.php to read real quotes from published authors.

Submit your manuscript here: http://www.dovepress.com/patient-preference-and-adherence-journal 